\title{
ANTIGEN SENSITIVITY EVALUATED BY TEAR-SPECIFIC AND SERUM-SPECIFIC IgE, SKIN TESTS, AND CONJUNCTIVAL AAND NASAL PROVOCATION TESTS IN PATIENTS WITH OCULAR ALLERGIC DISEASE
}

\author{
A. LEONARDI, M.C. BATTISTA, M. GISMONDI, I. A. FREGONA and A. G. SECCHI \\ Padua, Italy
}

\begin{abstract}
SUMMARY
The potential for ocular allergic patients to have a sitespecific antigen sensitisation was investigated using various diagnostic tests of allergen sensitivity in subjects with allergic conjunctivitis (AC: $n=135$ ), vernal keratoconjunctivitis (VK: $n=20$ ), rhinoconjunctivitis $(n=20)$ or rhinitis $(N=10)$. In the $\mathrm{AC}$ and VK patients, skin tests and conjunctival provocation tests (CPT) were performed, and the levels of specific $\mathrm{IgE}$ in serum and in tears were identified. A subgroup of 36 patients was also challenged with a nasal-specific provocation test (NPT). Results showed a poor correlation between skin test results and tear-specific IgE, and also between serumspecific IgE and tear-specific IgE in both $\mathrm{AC}$ and VK patients $(K<0.3)$. CPT and tear IgE were significantly correlated $(K=0.5)$ in the ocular allergic population. In patients with rhinoconjunctivitis or rhinitis, and in 10 normal subjects, results of CPT and NPT were in $100 \%$ agreement. Conversely, in patients with only conjunctivitis, little correlation was found between the results of CPT and NPT $(K=0.3)$. Tear-specific IgE was the only positive diagnostic sign of antigen sensitivity in $35 \%$ of VK patients and $30 \%$ of $\mathrm{AC}$ patients. These results suggest that the conjunctiva can be a uniquely sensitised target organ in allergic patients.
\end{abstract}

Conjunctival or keratoconjunctival allergic disease may at times be the only manifestation of allergen sensitivity presented by patients. While systemic tests can be useful in the diagnosis of ocular allergy, in a consistent percentage of cases of ocular allergy, tests such as the skin test and serum total and specific IgE are negative or not significant. ${ }^{1}$ In these patients the detection of specific IgE in tears and the response to specific conjunctival provocation tests

From: Department of Ophthalmology, Chair of Physiological Optics, University of Padua, Italy.

Correspondence to: Andrea Leonardi, MD, Via Palesa 27, I-35123 Padova, Italy. can provide a definitive diagnosis. ${ }^{2,3}$ High levels of specific IgE have been found in the tears of patients with negative systemic allergometric tests, suggesting that a uniquely ocular sensitisation, with local production of specific antibodies, ${ }^{3-9}$ may exist in some patients.

In an effort to investigate this phenomenon, patients with allergic conjunctivitis, vernal keratoconjunctivitis, rhinoconjunctivitis and rhinitis were subjected to different site-specific tests of allergic sensitisation, the results of which were statistically analysed for potentially significant correlations.

\section{Patients \\ MATERIALS AND METHODS}

This study involved 135 patients with allergic conjunctivitis, 20 patients with vernal keratoconjunctivitis, 20 patients with allergic rhinoconjunctivitis, and 10 patients with a history of allergic rhinitis only. All patients were in an inactive state of inflammation. Informed consent was obtained from all subjects involved in this study.

\section{Allergometric Tests}

All patients were challenged by skin test using the prick test procedure and the standard battery of pollens and perennial antigens. Tear samples were collected from patients, carefully avoiding irritation, using a microcapillary pipette positioned in the inferior conjunctival sac. Levels of specific IgE in serum and in tears were analysed using a fluorescence enzyme immunoassay (3-M Diagnostic System, Santa Clara, Calif., USA, distributed by Eurospital Pharma, Trieste, Italy).

Conjunctival provocation tests (CPT) were performed in 75 allergic conjunctivitis patients, again during an inactive phase of the allergic inflammation. Allergens were selected according to the clinical history of the patient or by skin testing, conjunctival or serum-specific IgE assay. The nasal mucosa or the conjunctiva was then challenged 
Table I. Correlation of antigen sensitivity identified by skin test and tear-specific IgE in patients with allergic conjunctivitis $(n=151$ paired analyses)

\begin{tabular}{lcc}
\hline & Tear IgE positive & Tear IgE negative \\
\hline Skin test positive & $65(43 \%)$ & $10(7 \%)$ \\
Skin test negative & $51(34 \%)$ & $25(16 \%)$ \\
\hline
\end{tabular}

The number of concordant and discordant test results of allergen sensitivity identified by skin test and tear-specific IgE in 135 patients (multiple allergens tested in some patients) with allergic conjunctivitis. Cohen's coefficient demonstrated no correlation $(K=0.19)$.

with one or more of the test positive allergens selected from the following: Graminaceae, Paritariae, Compositae, Alternariae, and/or Dermatophagoides (Bayropharm Italiana, Milan, Italy). Three concentrations of each allergen $(10,100,1000$ Rast Arbitrary Units/ml) were made from the stock solution at the time of testing. A positive CPT was defined by the presence of itching and/or tearing, and conjunctival redness and/or oedema. Additional CPTs were performed in the same patient with different antigens at weekly intervals.

Nasal provocation tests (NPT) were performed in 36 allergic patients ( 20 rhinoconjunctivitis, 10 rhinitis and 6 conjunctivitis) and in 10 normal subjects. For each patient the same panel of allergens was used for the CPT and nasal challenge. Increasing concentrations of allergen were inhaled at intervals of 20 minutes until a clinical reaction was observed.

\section{Statistics}

The correlation between the various diagnostic tests was determined using the agreement coefficient (Cohen's $K$ ). According to Fleiss, ${ }^{10}$ values below 0.40 signify no correlation between two variables, values greater than 0.75 represent a highly significant correlation, and values between 0.40 and 0.75 represent a fair to good correlation between two variables.

\section{RESULTS}

\section{Allergic Conjunctivitis Patients}

Of the 135 patients with active allergic conjunctivitis, 100 were positive to at least one of the allergometric tests performed. Because multiple allergens were tested in some patients, the comparison of skin test and tear-specific IgE results was possible in 151 paired analyses, and of tearand serum-specific IgE in 153 paired analyses.

Table II. Correlation of antigen sensitivity identified by serum specific IgE and tear-specific IgE in patients with allergic conjunctivitis $(n=153$ paired analyses)

\begin{tabular}{lcc}
\hline & Tear IgE positive & Tear IgE negative \\
\hline Serum IgE positive & $62(41 \%)$ & $8(5 \%)$ \\
Serum IgE negative & $51(33 \%)$ & $32(21 \%)$ \\
\hline
\end{tabular}

The number of concordant and discordant test results of allergen sensitivity identified by serum-specific $\operatorname{IgE}$ and tear-specific $\operatorname{IgE}$ in 135 patients (multiple allergens tested in some patients) with allergic conjunctivitis.

Cohen's coefficient demonstrated no correlation $(K=0.26)$.
Tear-Specific IgE Versus Skin Test Results. In the allergic conjunctivitis group, the skin test results and the presence of specific IgE in tears were in agreement in 90 of 151 cases (59\%): 65 (43\%) with positive test results in both and $25(16 \%)$ with negative test results in both (Table I). The remaining 61 cases (41\%) were discordant: 51 (34\%) were skin test negative/tear IgE positive and $10(7 \%)$ were skin test positive/tear IgE negative. Cohen's $K$ demonstrated a poor statistical correlation between the two tests $(K=0.19)$.

Serum-Specific Versus Tear-Specific IgE Test Results. The results of serum-specific IgE and tear-specific IgE showed concordance in 94 of 153 paired analyses $(62 \%)$ : $62(41 \%)$ with positive test results in both and $32(21 \%)$ with negative test results in both (Table II). Of the remaining 59 analyses (38\%), 51 of the cases $(33 \%)$ were tear $\operatorname{IgE}$ positive/serum IgE negative, and $8(5 \%)$ positive to serum IgE only. Cohen's coefficient showed a poor correlation between the two tests $(K=0.26)$.

Of the 100 patients who tested positively to at least one allergometric test, $30 \%$ tested positive only by specific IgE in tears.

\section{Vernal Conjunctivitis Patients}

Again, because multiple allergens were tested in some of the 20 vernal conjunctivitis patients, the sensitivity to allergens was identified by both skin test and tear-specific $\operatorname{IgE}$ in 37 cases and by both tear- and serum-specific IgE in 34 cases.

Tear-Specific IgE Versus Skin Test Results. There was no statistically significant correlation between skin test results and tear-specific IgE in patients with vernal conjunctivitis ( $K=0.17$ : Table III). Of the 37 paired analyses, $18(48 \%)$ were congruent: 6 positively $(16 \%)$ and 12 negatively (32\%). The remaining 19 cases $(51 \%)$ were inversely related, with all skin tests negative and tear-specific IgE positive.

Serum-Specific Versus Tear-Specific IgE Test Results. There was also no significant correlation between serumand tear-specific $\mathrm{IgE}$ in patients with vernal conjunctivitis $(K=0.23$ : Table IV). In 19 of the 34 paired analyses $(56 \%)$ there was concordance in the test results: 9 positively (27\%) and 10 negatively (29\%). Of the remaining 15 cases $(44 \%), 14(41 \%)$ were positive to tear-specific IgE but negative to serum IgE.

Of the 20 vernal conjunctivitis patients, $35 \%$ tested positively to allergen sensitivity only by the presence of specific IgE in tears.

Table III. Correlation of antigen sensitivity identified by skin test and tear-specific IgE in patients with vernal keratoconjunctivitis $(n=37$ paired analyses)

\begin{tabular}{lcc}
\hline & Tear IgE positive & Tear IgE negative \\
\hline Skin test positive & $6(16 \%)$ & 0 \\
Skin test negative & $19(51 \%)$ & $12(32 \%)$ \\
\hline
\end{tabular}

The number of concordant and discordant test results of allergen sensitivity identified by skin test and tear-specific IgE in 20 patients (multiple allergens tested in some patients) with vernal keratoconjunctivitis. Cohen's coefficient demonstrated no correlation $(K=0.17)$. 
Table IV. Correlation of antigen sensitivity identified by serum-specific IgE and tear-specific IgE in patients with vernal keratoconjunctivitis ( $n=34$ paired analyses)

\begin{tabular}{lcc}
\hline & Tear IgE positive & Tear IgE negative \\
\hline Serum IgE positive & $9(27 \%)$ & $1(3 \%)$ \\
Serum IgE negative & $14(41 \%)$ & $10(29 \%)$ \\
\hline
\end{tabular}

The number of concordant and discordant test results of allergen sensitivity identified by serum-specific IgE and tear-specific IgE in 20 patients (multiple allergens tested in some patients) with vernal keratoconjunctivitis.

Cohen's coefficient demonstrated no correlation $(K=0.23)$.

\section{Provocation Test Results in Allergic Subgroup}

Conjunctival provocation tests were performed in 82 allergic conjunctivitis patients who were also tested for the presence of the corresponding specific IgE in tears.

The correlation between specific conjunctival challenge and the presence of the same specific IgE antibodies in tears was significant $(K=0.54$ : Table V). In 71 of the 82 paired analyses (87\%) there was concordance of antigen sensitivity. However, in 6 sample pairs (7\%) CPT was positive in the absence of tear-specific IgE. Conversely, 5 sample pairs $(6 \%)$ had a negative response to CPT while positively demonstrating antibodies for that antigen in tears.

Conjunctival and nasal provocations with the same allergen were performed in a subgroup which contained 20 patients with allergic rhinoconjunctivitis, 10 patients with allergic rhinitis only, 6 patients with conjunctivitis only, and 10 normal subjects. CPT and NPT demonstrated $100 \%$ agreement in patients with rhinoconjunctivitis or rhinitis, and in normal controls ( $K=1.0$ : Table VI). However, in conjunctivitis patients with no nasal allergic history there was little agreement between the test results $(K=0.3)$.

\section{DISCUSSION}

The results of this study support the hypothesis that the conjunctiva can be the only sensitised tissue in patients with allergic disease. The poor correlation identified between systemic allergic sensitivity and local ocular sensitivity suggests that local tests such as tear-specific IgE and allergen challenge may be useful in the diagnosis of allergic conjunctivitis in patients with equivocal signs and symptoms. In fact, in $30 \%$ of allergic conjunctivitis patients and in $35 \%$ of vernal keratoconjunctivitis

Table V. Correlation of allergen sensitivity identified by the conjunctival provocation test (CPT) and tear-specific IgE in patients with allergic ocular disease ( $n=82$ paired analyses)

\begin{tabular}{lcc}
\hline & CPT positive & CPT negative \\
\hline Tear IgE positive & $62(76 \%)$ & $5(6 \%)$ \\
Tear IgE negative & $6(7 \%)$ & $9(11 \%)$ \\
\hline
\end{tabular}

The number of concordant and discordant test results of allergen sensitivity identified by a specific allergen conjunctival provocation test and tear-specific IgE in 75 patients (multiple allergens tested in some patients) with allergic conjunctivitis or vernal keratoconjunctivitis. Cohen's coefficient demonstrated a statistically significant correlation $(K=0.54)$. patients, only the local tests showed a positive allergen sensitivity.

Specific conjunctival challenge, was as expected, significantly correlated with the presence of specific IgE in tears $(87 \%)$. Discordant cases $(13 \%)$, in which the antibodies were present in tears but the patient had a negative clinical response to CPT, may have been due to a local down-regulation to allergen sensitivity. When the results to CPT were positive but specific IgE negative, the antibodies may have been present but completely bound to mast cells and not free in the tears.

To investigate further this potential exclusivity of sensitisation, some patients were challenged with the same allergen conjunctivally and nasally. Results showed a poor correlation between NPT and CPT in patients with only allergic conjunctivits. This selective sensitisation to environmental allergens may be due to anatomical and physiological differences between the nasal and conjunctival mucosa.

If the origin of specific antibodies in tears is, indeed, local production, local modulation of the immune system is a possible treatment for ocular allergic disease. It seems unlikely that tear IgE originates solely from serum IgE, given that it is possible to have only tear-specific or only serum-specific IgE, and also that, in this study, the tearspecific IgE was often higher than serum-specific $\operatorname{IgE}$ in ocular allergic patients. It is possible that the increase in specific antibodies in tears is due to the increased number of conjunctival epithelial mast cells observed in patients with allergic ocular disease. ${ }^{11-13}$ Additionally, local sensitisation and production of IgE may occur by allergen activation of antigen-presenting cells and lymphocytes in the conjunctiva, causing them to migrate to the regional lymph nodes, activating specific $\mathrm{T}$ and $\mathrm{B}$ cells which can then 'home back' to the conjunctiva and produce locally specific IgE. ${ }^{14-16}$

In conclusion, this study demonstrates the poor correlation between local and systemic allergometric tests in patients with allergic conjunctivitis and vernal keratocon-

Table VI. Correlation of allergen sensitivity identified by the conjunctival provocation test (CPT) and the nasal provocation test (NPT) $(n=46$ paired analyses $)$

\begin{tabular}{lcc}
\hline Patient population & $\begin{array}{c}\text { CPT positive } \\
(\%)\end{array}$ & $\begin{array}{c}\text { NPT positive } \\
(\%)\end{array}$ \\
\hline $\begin{array}{l}\text { Rhinoconjunctivitis }(n=20) \\
K=1.0^{*}\end{array}$ & 100 & 100 \\
$\begin{array}{l}\text { Rhinitis }(n=10) \\
K=1.0^{*}\end{array}$ & 100 & 100 \\
$\begin{array}{l}\text { Conjunctivitis }(n=6) \\
K=0.3^{* *}\end{array}$ & 100 & 50 \\
$\begin{array}{l}\text { Normal subjects }(n=10) \\
K=1.0^{*}\end{array}$ & 0 & 0 \\
\hline
\end{tabular}

The number of concordant and discordant test results of allergen sensitivity identified by specific allergen conjunctival provocation test and specific allergen nasal provocation test in 46 patients with various allergic histories.

* Cohen's coefficient demonstrated a statistically significant correlation

$(K=1.0)$.

** Cohen's coefficient demonstrated no correlation $(k=0.3)$. 
junctivitis. The high percentage of patients with specific antibodies only in tears, and the high correlation between the specific conjunctival provocation test and specific tear IgE support the hypothesis that an exclusively local immunodeviation can result in allergic conjunctivitis. The aim of further studies is to modulate this local phenomenon with specific local immunotherapy.

Key words: Allergic conjunctivitis, Allergometric tests, IgE, Vernal keratoconjunctivitis.

\section{REFERENCES}

1. Leonardi A, Fregona IA, Gismondi M, Daniotti E, Carniel G, Secchi AG. Correlation between conjunctival provocation test (CPT) and systemic allergometric tests in allergic conjunctivitis. Eye 1990;4:760-4.

2. Leonardi A, Daniotti E, Carniel G, DeCarli M, Gismondi M, Fregona IA, Secchi AG. I test allergometrici sistemici e lacrimali nella diagnosi di congiuntivite allergica. In: Proceedings of the 69th Congress of the SOI. ESAM, Rome: Futura Publishing, 1990:329-34.

3. Secchi AG, Leonardi A, Daniotti E, Camiel G, Fregona IA. Tear specific IgE in the diagnosis of allergic hay fever type conjunctivitis. In: Usui M, Ohno S, Aoki K, editors. Ocular immunology today. Amsterdam: Elsevier, 1990:29-32.

4. Allansmith MR, Hahn GS, Simon MA. Tissue, tear and serum IgE concentrations in vernal conjunctivitis. Am J Ophthalmol 1976;81:506-11.

5. Ballow M, Mendelson L. Specific immunoglobulin E antibodies in tear secretions of patients with vernal conjunctivitis. J Allergy Clin Immunol 1980;66:112-8.

6. Ballow M, Mendelson L, Donshik P, Rooklin A, Rapacz P. Pollen specific IgE antibodies in tears of patients with aller- gic-like conjunctivitis. J Allergy Clin Immunol 1984;73: 376-80.

7. Sompolinsky D, Samara Z, Zavaro A, Barishak Y. Allergenspecific immunoglobulin $\mathrm{E}$ antibodies in tears and serum of vernal conjunctivitis patients. Int Arch Allergy Appl Immunol 1984;75:317-21.

8. Kari O, Salo OP, Bjoeksten F, Backman A. Allergic conjunctivitis, total and specific IgE in the tear fluid. Acta Ophthalmol (Copenh) 1985;63:97-9.

9. Liotet S, Warnet VN, Arrata M. Lacrimal immunoglobulin E and allergic conjunctivitis. Ophthalmologica 1986;186: $31-4$.

10. Fleiss JL. Statistical methods for rates and proportions, 2nd ed. New York: Wiley, 1981.

11. Allansmith MR, Baird KS, Greiner JV. Vernal conjunctivitis and contact-lens associated giant papillary conjunctivitis. Invest Ophthalmol Vis Sci 1988;29(Suppl):269

12. Butrus S, Irani AA, Tabbara KF, Schwartz LB. Mast cell subtypes in vernal and in giant papillary conjunctivitis. Invest Ophthalmol Vis Sci 1988;29(Suppl):269.

13. Morgan SJ, Williams JH, Walls AF, Church MK, Holgate ST, McGill JI. Mast cell number and staining characteristics in the normal and allergic conjunctiva. J Allergy Clin Immunol 1991;87:111-6.

14. Strober W, James SP. The mucosal immunosystem. In: Stites DP, Terr Al, editors. Basic and clinical immunology, London: Prentice-Hall, 1991:175-86.

15. Easty DL, Birkenshaw M, Merrett T, Merrett J, Entwhistle C, Amer B. Immunological investigations in vernal eye disease. Trans Ophthalmol Soc UK 1980;100:98-107.

16. Abu EL, Asrar AM, Van der Oord JJ, Goeboes K, Missotten L, Emarah MH, Desmet V. Immunopathological study of vernal keratoconjunctivitis. Graefes Arch Clin Exp Ophthalmol 1989;227:374-9. 